\title{
The most complete apoptotic saga
}

\author{
Cell Death and Differentiation (2007) 14, 1068-1069. doi:10.1038/sj.cdd.4402113
}

Apoptosis and Cancer Therapy: From Cutting-edge Science to Novel Therapeutic Concepts, 2 Volumes. Edited by K-M Debatin and S Fulda. Wiley-VCH Verlag GmbH \& Co. KGaA, Weinheim, Germany, 2006. 1160 pp. ISBN 3-527-31237-4. $£ 310.00 / 465.00 / \$ 610.00$, hardcover

The two-volume book on Apoptosis and Cancer Therapy comprising 43 chapters is written by well-known scientists from most of the major centers engaged in cell death research. Roughly one-third of the contributors are from Germany. This is not surprising since both editors are from the University of Ulm, and since the German school (C Vogt, R Virchow, A Weismann, L Stieda, A Goette, C Weigert, J Cohnheim, W Flemming, S Mayer, F Nissen, D Barfurth, F von Recklinghausen, L Gräper, A Glücksmann, etc.) has been recognized worldwide for the pioneer studies elaborating the concept of cell death.

In the first part, the authors discuss in depth the current knowledge on receptor-mediated cell death triggering by positive signals (extracellular death ligands) or by negative signals (lack of ligands). Importantly, the potential role(s) of these unique cell surface sensors in cancer development and progression are considered. The intrinsic apoptosis signaling linked to mitochondria is the focus of the second part. It begins with a deep analysis of mitochondrial proteins realizing their apoptogenic function via caspase-dependent or caspaseindependent routes upon the release into the cytosol. The following chapters deal with the modes of HtrA2/Omi actions and with a comprehensive discussion of pro- and antideath effects of AIF. Of considerable interest is the recent finding that this multifunctional protein might act as a strong death inducer preferentially under hypoxic conditions.

Part III is dedicated to the analysis of cell death effector systems, with particular reference to their role in oncogenesis and resistance to chemotherapy. The current views on biochemical mechanisms of caspase activation, the modulators of apoptosome formation and function as well as cancerrelated defects in caspase-dependent apoptosis signaling are amply treated. Of special importance are the recent data from the large-scale analysis using caspase substrate prediction software suggesting that roughly 4500 proteins are potential intracellular targets of caspases. Since the number of the already known caspase substrates is about 500 , it may be expected that many non-apoptotic functions of these versatile proteases remain to be identified.

In part IV the discussion on the role of mitochondria in apoptosis continues with particular emphasis on the balance between pro- and antiapoptotic members of $\mathrm{Bcl}-2$ family. Proapoptotic Bcl-2 family members Bax, Bak and Bid are reviewed, with a concluding chapter on $\mathrm{BH}$-only proteins, Puma and Noxa. In the next section, the inhibitors of apoptosis proteins (IAPs) are considered with emphasis on recent advances in our understanding of the physiology and pathophysiology of XIAP and survivin. From the therapeutic standpoint, survivin is a most promising target for future therapy since according to the author's statement, 'no evidence of tumor resistance for survivin-based therapy has been reported.'

In part $\mathrm{VI}$ the reader is acquainted with the control mechanisms of cell survival focusing on anti-death signaling mediated by NF-KB, PI3K, PKB/Akt, Ras, and Raf, especially newly discovered pathways involving their modulators. For instance, c-IAP1 and c-IAP2 proteins that inhibit (together with TRAF-1 and -2) caspase-8 and TNF-mediated apoptosis without binding to caspase- 8 are considered. The data on the novel regulators of PKB activity (ATM, PHLPP) and recently identified PKB substrates (e.g., YES-associated protein) are also presented.

Part VII concerns the crucial role of oncogenes and tumor suppressor genes such as $p 53$, the $p 53$-related gene $p 73$ and $R b$ in regulation of cell cycle progression and apoptosis. Of particular relevance is the discussion on the mechanisms of nontranscriptional p53-mediated apoptosis, the models for antiapoptotic functions of p53 or Rb, and the tumorigenic potential of $\Delta \mathrm{Np} 73$. The useful section that follows serves to present current knowledge on the participation of calcium ions in the stress response including apoptotic or necrotic cell death. Novel details of apoptosis signaling by endoplasmic reticulum-mitochondrial fluxes of $\mathrm{Ca}^{2+}$ as well as crosstalk between caspases, calpains and other proteases in regulation of cell death are considered.

In part IX, the mechanisms of alternative, caspaseindependent cell death pathway involving the release of cathepsin proteases from lysosomes are presented. As the authors state, apart from caspase-independent cell death, lysosomal membrane permeabilization may trigger caspase activation and subsequent apoptosis. Although the precise mechanism of this sequence of events requires further elucidation, the prospects for targeting lysosomes for cancer treatment are intriguing.

The recognition and subsequent engulfment of dying and/or dead cells by phagocytic cells are considered in two final chapters of volume 1 . The authors conclude with some paradoxical speculations about possible involvement of apoptosis in promoting oncogenesis due to providing key stimuli for infiltration of premalignant foci by macro- 
phages, which in turn promote tumor cell survival as well as suppress antitumor immunity. Nevertheless, this hypothesis does not seem to take into account other components of the immune system being of importance in an antitumor response.

The second volume of the book begins with a chapter on genetically engineered mouse models. The authors analyze in details the achievements and pitfalls pertaining to transgenic, knockout, knock-in and knock-down animals. The relation between apoptosis and chemoresistance is discussed within this context, providing evidence that such mouse models can be utilized to screen for gene activities that may confer resistance.

Part XII describes modern strategies of noninvasive imaging of cancer biomarkers as well as visualization of apoptotic cells in vivo. The real-time techniques such as MRI, MRS and ultrasound imaging are under development today. The next important topic of this section is the design of microarray technologies for identifying molecular changes involved in oncogenesis.

Part XIII consists of four chapters discussing cellular stress, DNA damage and repair. Its prelude is the analysis of the apoptotic pathways triggered by various cellular stressors, followed by discussion of stress signaling mediated by $\mathrm{BH}$ - only proteins, SAPKs, HSPs and NF- $\kappa \mathrm{B}$, and the ability of cancer cells to survive in the hypoxic environment. The rest of the section is devoted to the major DNA repair signaling mechanisms. It is evident that the genomic instability due to defective DNA repair, which strongly correlates with the apoptotic response (as nicely reviewed in chapter 31), is a risk factor predisposing for many types of neoplasia.

The closing part includes a comprehensive list of different ways of modulating apoptosis in cancer therapy. Unfortunately, apoptosis induction of tumor cells by antiangiogenic drugs due to the blockage of nutrient supply and waste removal, or the death of endothelial cells after metronomic low-dose chemotherapy seems to have escaped the authors' attention.

All in all, I would strongly recommend this rather encyclopedic book for anyone who is interested in cell death research, particularly in manipulation of apoptosis for the development of therapeutic strategies in cancer treatment.

\section{A Philchenkov}

RE Kavetsky Institute of Experimental Pathology, Oncology and Radiobiology, 45 Vasilkovskaya Street, Kyiv 03022, Ukraine

E-mail: a_philch@onconet.kiev.ua 